\title{
Effects of Intracerebroventricular Sugammadex Administration on Central Nervous System in Rats
}

Hale Aksu Erdost*, Elvan Ocmen, Seden Duru, Burc Aydın and Ali Necati Gokmen

Faculty of Medicine, Dokuz Eylül University, Izmir, Turkey

\begin{abstract}
Introduction: Sugammadex (Bridon $®$ ) (SUG) is a recently developed neuromuscular block reversing agent. SUG can reverse also deep neuromuscular blockages in a short time period unlike other existing agents. SUG passes across blood brain barrier (BBB) in a very low ratio in normal patients. However SUG may pass the BBB in a higher ratio in patients whom BBB integrity is decreased. Since SUG passes BBB in a low ratio in normal patients there are only a small amount of studies investigating effects of this agent on central nervous system (CNS). In this study we aimed to assess the effects of SUG administered directly to intracerebroventricular space on CNS system of rats.
\end{abstract}

Materials and Method: A total of 36 Wistar-Albino rats with normal motor activity weighting between 250-280 g were included in this study. Anesthesia was achieved with intraperitoneal $50 \mathrm{mg} / \mathrm{kg}$ sodium thiopental. The rats were divided into 6 equal groups randomly as one group being the control group. The experiment groups were received $2,4,8,16$ and $32 \mathrm{mg} / \mathrm{kg}$ sugammadex via intracerebroventricular cannula. Effects of the SUG on CNS were assessed based on a 5 point scale.

Results: Intracerebroventricular SUG administration did not result in any changes in behavioral status, locomotor activity or posture at any doses $(2,4,8,16$ and $32 \mathrm{mg} / \mathrm{kg})$. There was no tonic clonic convulsion or seizure development following the sugammadex administration.

Discussion: SUG barely passes the BBB in normal patients. However it was stated that this drug can pass BBB in higher ratios in certain patients. Therefore investigating the effects of SUG on CNS is an emerging subject of experiments. In our study we could not find any adverse effect of SUG on CNS even at high doses administered directly to intracerebroventricular space. However presence of a study indicating an increase in apoptotic cell death in cell cultures in presence of SUG makes it difficult to make a statement that SUG does not have any adverse effect on CNS. The authors of the aforementioned study stated a connection between decrease in cholesterol levels and apoptosis. It can be speculated that some mechanisms in live animals may restore this decrease in cholesterol levels occurring in presence of SUG therefore prevents the cells from apoptosis.

Conclusion: In our study SUG did not cause any adverse effect on CNS in rats. Further studies assessing the relationship between SUG and cholesterol control mechanisms in neurons are necessary in order to make a certain statement.

Keywords: Neuromuscular block; CNS; Sugammadex

\section{Introduction}

Sugammadex (Bridon ${ }^{\circ}$ ) (SUG) is a recently developed neuromuscular block reversing agent. SUG can reverse also deep neuromuscular blockages in a short time period unlike other existing agents $[1,2]$. In a recent study, it was observed that administration of SUG via intravenous (IV) route does not cause any alteration in antiepileptic drug levels. It was stated that SUG does not interact with antiepileptic drugs [3]. There are only limited numbers of studies in the literature about this routinely used new drug. SUG passes across blood brain barrier (BBB) in a very low ratio in normal patients. However SUG may pass the BBB in a higher ratio in patients whom $\mathrm{BBB}$ integrity is damaged like $\mathrm{Al}$ zheimer [4], Parkinson [5], multiple sclerosis [6], disease associated neurodegeneration, traumatic brain/spinal cord injury [7], ischemia [8], meningitis [9] or immature nervous system [10]. Since only a small amount of SUG passes the BBB there are a few studies investigating the toxic effects of SUG on central nervous system. In this study we aimed to assess the effects of SUG administered directly to intracerebroventricular space on central nervous system (CNS).

Cyclodextrins are cyclic oligosaccharides consisting of six or more $\alpha-1,4$ linked D-glucopyranose units. They also contain a large number of hydroxyl groups (Figure 1). Their cyclic structure creates a cavity which is water soluble hydrophilic at the exterior and hydrophobic at the interior. The number of glucopyranose molecules determines the size of the cavity. A structure consisting of six, seven and eight glucopyranoses are called as a, b and c-cyclodextrins respectively. A number of glucopyranose below six cannot form a cyclodextrin because of steric hindrances. Also a cyclodextrin with 9 or more glucopyranose molecules is unstable and their cavity is collapsed thus they can contain smaller molecules. The cavity size of $\mathrm{a}, \mathrm{b}$ and $\mathrm{c}$-cyclodextrins are ranged between $4.7-5.3 \mathrm{~A}^{\circ}, 6.0-6.5 \mathrm{~A}^{\circ}$ and $7.5-8.3 \mathrm{~A}^{\circ}$ respectively. The size of the cavity diameter determines the molecules that can be encapsulated [11].

Sugammadex is a modified $\gamma$-cyclodextrin specifically designed to encapsulate rocuronium and chemically similar aminosteroidal muscle relaxants such as vecuronium [12]. The mechanism of action is completely different from acetylcholine esterase inhibitors. Sugammadex binds to free rocuronium molecules in plasma nearly immediately after administration thus results in decrease in plasma levels of rocuronium. This creates a gradient of rocuronium between

*Corresponding author: Hale Aksu Erdost, Faculty of Medicine, Dokuz Eylül University, Izmir, Turkey, Tel: +905323615722; E-mail: hale.erdost@deu.edu.tr

Received February 13, 2015; Accepted January 11, 2016; Published January 16, 2016

Citation: Erdost HA, Ocmen E, Duru S, Aydın B, Gokmen AN (2016) Effects of Intracerebroventricular Sugammadex Administration on Central Nervous System in Rats. Brain Disord Ther 5: 206. doi:10.4172/2168-975X.1000206

Copyright: $\odot 2016$ Erdost HA, et al. This is an open-access article distributed under the terms of the Creative Commons Attribution License, which permits unrestricted use, distribution, and reproduction in any medium, provided the original author and source are credited. 
tissue and plasma and rocuronium molecules in the tissues start to move to plasma where they also get encapsulated by free SUG molecules [13]. Then the complex is filtered by the glomerulus and eliminated through the kidney.

SUG is a highly selective binding agent for aminosteroidal neuromuscular blockers (NMB). SUG encapsulates the complete steroid part of the molecule strongly. As it was also mentioned above a concentration gradient develops that moves the NMB agent to central compartment by diffusion. This process frees the acetylcholine receptors and allows acetylcholine to bind again. As a result; total plasma concentration of rocuronium increases and it was found that this increase is parallel to recovery of muscle contractions. [14]. It was demonstrated that the SUG-ROC complex is rapidly excreted via kidneys [15]. Since the SUG does not affect the acetylcholine receptor or the acetylcholine amount present at the nicotinic or muscarinic receptor any adverse effect related to these receptors is not expected.

Other commonly used neuromuscular blockage reversing agents are neostigmine and edrophonium. However these agents have also some handicaps. These agents both need to use in association with atropine or glycopyrrolate to avoid side effects like tachycardia and hypotension. Therefore use of neostigmine may be contraindicated in patients with severe asthma or cardiovascular disease. Another limitation of use of this agent is its relatively slow onset of action. Increasing the administered dose is not always a solution because it exhibits ceiling effect greater than $70 \mu \mathrm{g} / \mathrm{kg}$ dose due to limited amount of acetylcholine at the neuromuscular junction. Additionally the recovery of neuromuscular blockage following neostigmine administration is not fast enough [16].

The adverse effects caused by commonly used NMB reversing agents bring SUG forward among other agents. However effects of SUG on CNS are still not well known. It was asserted that SUG can pass the BBB in higher ratios in patients with damaged $\mathrm{BBB}$ integrity compared to normal individuals. We aimed to investigate the effects of SUG on CNS.

\section{Materials and Method}

This study was carried out at Dokuz Eylül University Medical Faculty (DEUMF) with approval of Ethical Committee of Animal Experiments. A total of 36 Wistar-Albino rats with normal motor activity weighting between $250-280 \mathrm{~g}$ were included in this study. The rats sex were female, all were $87 \%$ homogeneous, were aged $18-24$ months, and exhibited normal activity.

Anesthesia phase was performed with $50 \mathrm{mg} / \mathrm{kg}$ sodium thiopental administered via intraperitoneal route. The animals were placed on the stereotaxi device (Rodent Stereotaxic Instruments ${ }^{\oplus}$, Harvard Apparatus, USA) after the anesthesia (Figure 1). Lateral ventricle entrance stereotaxic points were determined as $1.6 \mathrm{~mm}$ lateral and

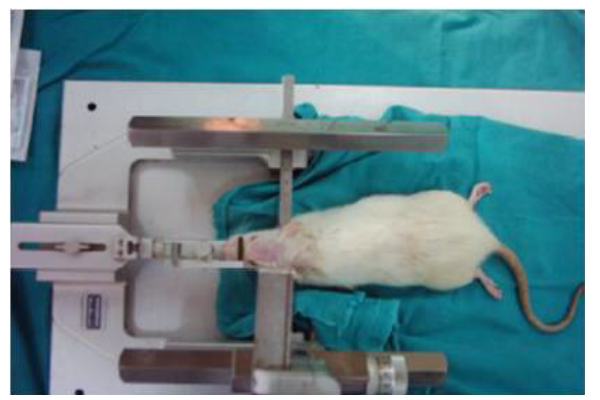

Figure 1: The animals were placed on the stereotaxi device after the anesthesia.

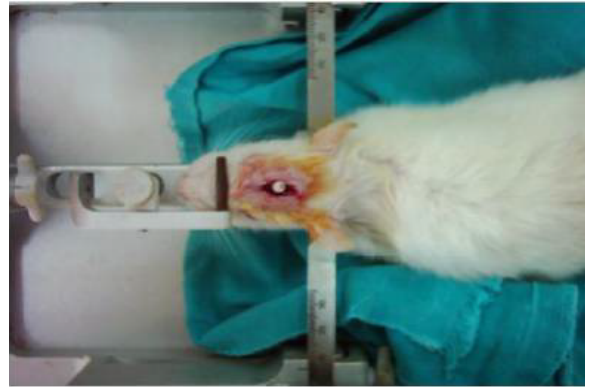

Figure 2: Lateral ventricle was entered via intracerebroventricular cannulas and appropriate probes.

$0.9 \mathrm{~mm}$ to caudal to bregma [17]. Lateral ventricle was entered via intracerebroventricular cannulas and appropriate probes (Figure 2). It was confirmed with cerebrospinal fluid flow. After this intervention it was waited for 48 hours for rats to return to their usual behaviors and rats were divided into 6 groups randomly.

Control Group (CG, n:6) A total of $50 \mu$ l saline solution was administered as $5 \mu$ l of equal doses to each rat via intracerebroventricular (ICV) cannula.

Group 1 (G1) (n:6) $2 \mathrm{mg} / \mathrm{kg}$ SUG was administered via ICV cannula repeatedly.

Group 2 (G2) (n:6) 4 mg/kg SUG was administered via ICV cannula repeatedly.

Group 3 (G3) (n:6) $8 \mathrm{mg} / \mathrm{kg}$ SUG was administered via ICV cannula repeatedly.

Group 4 (G4) (n:6) 16 mg/kg SUG was administered via ICV cannula repeatedly.

Group 5 (G5) (n:6) $32 \mathrm{mg} / \mathrm{kg}$ SUG was administered via ICV cannula repeatedly.

The volume of the total fluid that administered to rats in all experimental groups was equal to that administered to control group.

\section{Assessment of the effects of SUG administered via ICV route was based on a 5 point scale}

$0=$ no observable effects

$1=$ decreased locomotor activity and/or piloerection

$2=$ agitation or shivering

$3=$ entire body tremors, posturing, or splayed limbs

$4=$ tonic-clonic convulsions or seizures

After the administration of the drug via ICV cannula rats were placed in their cages (one in each cage) and they were assessed for 2 hours for any motor or behavioral changes with a 5 point scale. The behavior scala was used by Szenohradszky et al. [18] for observing the central nervous system effects of muscle relaxants in rats.

Methylene blue was administered via ICV cannula before the experiment is ended in for postmortem control of the methylene blue if it is in ICV space or not (Figure 3).

Statistical analysis was performed with Statistical Package of Social Sciences 15 (SPSS 15.0, Chicago, IL, USA). $\mathrm{p}<0.05$ was considered as statistically significant. 


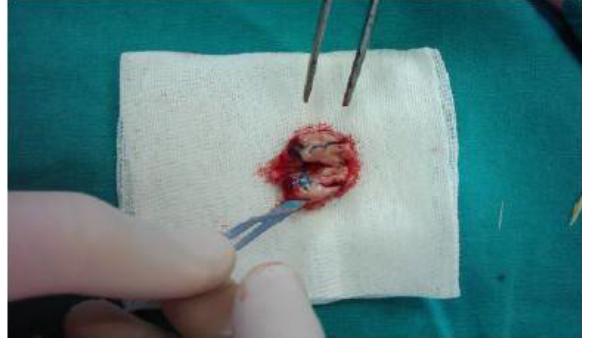

Figure 3: Methylene blue was administered via ICV cannula before the experiment is ended in for postmortem control of the methylene blue if it is in ICV space or not.

\section{Results}

Intracerebroventricular SUG administration did not result in any changes in behavioral status, loco motor activity or posture at any doses $(2,4,8,16$ and $32 \mathrm{mg} / \mathrm{kg})$. We used the particular doses because the recommended dosage of the drug is $2,4,8,16$ and $32 \mathrm{mg} / \mathrm{kg}$ in the clinic.

There was no tonic clonic convulsion or seizure development following the sugammadex administration. All of the rats survived the experiment. All the rats in each group were assessed as 0 point according to the behavior scale. There were no statistically significant difference between groups ( $\mathrm{p}>0.99$ )

After experiment ended, all rats had a metilen blue injection through the ICV cannula and with high dose anesthetic rats sacrificed. Post mortem brain dissection was performed with all rats and confirmed the metilen blue was placed in the intracerebroventricle.

\section{Discussion}

NMB agents are widely used during surgery in order to facilitate the endotracheal intubation, artificial ventilation and to provide the surgeon an easier work field [19]. The SUG binds with amino-steroid molecule with its lipophilic core and makes the molecule unable to bind acetylcholine receptor at the neuromuscular junction [20]. With this isolated and unique action mechanism of the SUG, autonomic instabilities of anti-cholinesterase drugs such as neostigmine are avoided. Furthermore; anti-muscarinic agents like atropine do not need to be co-administered which are contraindicated to use in certain patient groups like cardiopulmonary disease or asthma patients.

One of the most commonly used muscle relaxants for general anesthesia is rocuronium (ROC) and vecuronium (VEC) due to their relatively decreased side effects. ROC has a reasonably rapid onset at high doses and it can be used as first choice instead of suxamethonium when a quick and short term muscle relaxation is needed because suxamethonium has some serious side effects like anaphylaxis, increasing serum potassium levels and different cardiovascular responses. Since the effects of ROC can be reversed with SUG rapidly; this agent can also be used for short term muscle relaxations [21]. It can also be challenging to restore the effects of muscle relaxants and this can cause cannot intubate-cannot ventilate situation in some patient groups. Therefore use of sugammadex in these patient groups is the best choice to reverse the effects of ROC and VEC. SUG binds to ROC or VEC with a ratio of 1:1. Use of SUG $n$ reverse moderate or deep neuromuscular blockage depending on the dose. However there are still some questions remaining unanswered about this agent. Since SUG is a considerably new agent using for reversal of effects of neuromuscular blockage agents some questions still remaining about its side effects. It was stated that SUG penetrates the blood brain barrier (BBB) in a low ratio $(<3 \%)$ due to its singular architecture, charge and high molecular weight (2178.01) [22]. According to US FDA briefing document [23] and European Medicines Agency scientific document (EMEA) [24] prepared based on conventional studies of safety pharmacology, repeated dose toxicity, genotoxicity and toxicity to reproduction, local toxicity or compatibility with blood there is no hazardous properties of this agent. There are some controversies about potential renal and lung toxicities on high doses. The side effects of SUG concerning central nervous system are not clear and it is still a question if SUG causes toxicity on central nervous system.

In a study of Palanca it was stated that SUG causes neuronal apoptosis in primary cultures [25]. Primary cultures of cortical neurons were prepared from the cerebral cortex of 14-15 day old rat fetuses (5 female rats to obtain fetuses). Apoptotic and necrotic cell death induced by SUG were distinguished by using fluorescence microscopy [26]. 75 $\mu \mathrm{g} / \mathrm{ml}$ concentration of SUG was used in the experiment. They stated that this concentration of SUG causes neuronal damage. $90 \%$ of cell death caused by apoptosis however remaining $10 \%$ of death was caused by necrosis. They indicated that SUG caused apoptosis on a statistically significant level after 6,12 and 24 hours of SUG $75 \mu \mathrm{g} / \mathrm{ml}$ exposure. They also indicated that a statistically significant cell death was observed after 24 hours of SUG $37.5 \mu \mathrm{g} / \mathrm{ml}$ exposure. They further investigated the neuronal cholesterol levels in case of SUG presence whether neuronal apoptosis is caused due to decrease in neuronal cholesterol levels. They found that SUG causes decrease in membraneassociated, cytosolic and mitochondrial cholesterol levels. Moreover they indicated that cholesterol enrichment prevented the release of different pro-apoptotic molecular signals (including CytC, AIF, Smac/ Diablo or CASP-3 activity) caused by SUG. Therefore they indicated a relationship between apoptosis activation induced by SUG addition to neuronal cells and neuronal cholesterol homeostasis.

Because sugammadex does not act as neostigmine or edrophonium, by inhibition of acetylcholinesterase and indirect action on receptors, but by encapsulation in the plasma, it is not expected to have such side effects than anticholinesterase agents. Most of the related side effects observed in phase II and III studies were unspecific including hypotension, movement coughing, dry mouth or nausea. Prolongation of the corrected QT interval have been described but with the same rate than in the placebo group. This can be observed with several anesthetic agents; therefore its signification was highly questionable. Fuchs-Buder et al. have confirmed the lack of cardiovascular effects of both 2 and 4 $\mathrm{mg} / \mathrm{kg}$ sugammadex in patients with cardiovascular disease undergoing non cardiac surgery [16].

\section{Conclusion}

The SUG was introduced as a miracle synthetic drug at first. Since this drug barely passes the BBB there is no adverse effect described in the literature specific to central nervous system. However SUG can pass $\mathrm{BBB}$ in higher amounts in patients with decreased BBB integrity. In our study we could not find any adverse effect of SUG on CNS even at high doses administered directly to intracerebroventricular space. However presence of a study indicating an increase in apoptotic cell death in cell cultures in presence of SUG makes it difficult to make a statement that SUG does not have any adverse effect on CNS. The authors of the aforementioned study stated a connection between decrease in cholesterol levels and apoptosis. It can be speculated that some mechanisms in live animals may restore this decrease in cholesterol levels occurring in presence of SUG therefore prevents the cells from apoptosis. Limitations of the study were first of all we just performed very simple behavioral assessment. There might be some 
Citation: Erdost HA, Ocmen E, Duru S, Aydın B, Gokmen AN (2016) Effects of Intracerebroventricular Sugammadex Administration on Central Nervous System in Rats. Brain Disord Ther 5: 206. doi:10.4172/2168-975X.1000206

behavioral changes that couldn't be checked.And secondly we didn't investigate anatomical or physiological effects after drug treatments. Further studies assessing these relationships are necessary in order to make a certain decision.

\section{References}

1. Naguib M (2007) Sugammadex: another milestone in clinical neuromuscular pharmacology. Anesth Analg 104: 575-581.

2. Chambers D, Paulden M, Paton F, Heirs M, Duffy S, et al. (2010) Sugammadex for the reversal of muscle relaxation in general anaesthesia: a systematic review and economic assessment. Health Technol Assess 14: 1-211.

3. Yusuke K, Miyake N, Nakayama H, Yogo H, Ozaki M, et al. (2011) Effect of Sugammadex on Plasma Concentration of Anti-epileptic Drugs. ASA Annual Meeting, USA.

4. Bell RD, Zlokovic BV (2009) Neurovascular mechanisms and blood-brain barrier disorder in Alzheimer's disease. Acta Neuropathol 118: 103-113.

5. Stolp HB, Dziegielewska KM (2009) Review: Role of developmental inflammation and blood-brain barrier dysfunction in neurodevelopmental and neurodegenerative diseases. Neuropathol Appl Neurobiol 35: 132-146.

6. Waubant E (2006) Biomarkers indicative of blood-brain barrier disruption in multiple sclerosis. Dis Markers 22: 235-244.

7. Shlosberg D, Benifla M, Kaufer D, Friedman A (2010) Blood-brain barrier breakdown as a therapeutic target in traumatic brain injury. Nat Rev Neurol 6: 393-403.

8. Kaur C, Ling EA (2008) Blood brain barrier in hypoxic-ischemic conditions. Curr Neurovasc Res 5: 71-81.

9. Mook-Kanamori BB, Geldhoff M, van der Poll T, van de Beek D (2011) Pathogenesis and pathophysiology of pneumococcal meningitis. Clin Microbiol Rev 24: 557-591.

10. Saunders NR, Knott GW, Dziegielewska KM (2000) Barriers in the immature brain. Cell Mol Neurobiol. 20: 29-40.

11. Booij LH (2009) Cyclodextrins and the emergence of sugammadex. Anaesthesia 64 Suppl 1: 31-37.

12. Zhang MQ (2003) Drug-specific cyclodextrins: the future of rapid neuromuscular block reversal? Drugs Future 28: 347-354

13. Bom A, Bradley M, Cameron K, Clark JK, Van Egmond J, et al. (2002) A novel concept of reversing neuromuscular block: chemical encapsulation of rocuronium bromide by a cyclodextrin-based synthetic host. Angew Chem Int Ed Engl 41: 266-270.
14. Epemolu O, Bom A, Hope F, Mason R (2003) Reversal of neuromuscular blockade and simultaneous increase in plasma rocuronium concentration after the intravenous infusion of the novel reversal agent Org 25989. Anesthesiology 99: 632-637.

15. Epemolu O, Mayer I, Hope F, Scullion P, Desmond P (2002) Liquid chromatography/mass spectrometric bioanalysis of a modified c-cyclodextrin (Org 25969) and rocuronium bromide (Org 9426) in guinea pig plasma and urine: its application to determine the plasma pharmacokinetics of Org 25969 Rapid Communications in Mass Spectrometry 16: 1946-1952.

16. Fuchs-Buder T, Meistelman C, Raft J (2013) Sugammadex: clinical development and practical use. Korean J Anesthesiol 65: 495-500.

17. Baykal M (2007) Ratlarda Intraserebroventriküler Verilen Rokuronyumun Santral Sinir Sistemi Üzerine Etkileri ve Epileptik Dozunun Arastirilmasi Tez Çalismasi Izmir.

18. Szenohradszky J, Trevor AJ, Bickler P, Caldwell JE, Sharma ML, et al. (1993) Central nervous system effects of intrathecal muscle relaxants in rats. Anesth Analg 76: 1304-1309.

19. Claudius C, Garvey LH, Viby-Mogensen J (2009) The undesirable effects of neuromuscular blocking drugs. Anaesthesia 64(1): 10-21.

20. Adam JM, Bennett DJ, Bom A, Clark JK, Feilden H, et al. (2002) Cyclodextrinderived host molecules as reversal agents for the neuromuscular blocker rocuronium bromide: synthesis and structure-activity relationships. J Med Chem 45: 1806-1816.

21. Yang LP, Keam SJ (2009) Sugammadex: a review of its use in anaesthetic practice. Drugs 69: 919-942.

22. Farrar JT (2008) Meeting of the Anesthetic and Life Support Drugs FDA AdvisoryCommittee, Silver Spring, MD. Sugammadex 222-225.

23. Food and Drug Administration. Anesthetic and Life Support Advisory Committee Meeting: briefing document (background package) for sugammadex sodium injection.

24. European Medicines Agency. European Public Assessment Report for Bridion (sugammadex): scientific discussion.

25. Palanca JM, Aguirre-Rueda D, Granell MV, Aldasoro M, Garcia A, et al. (2013) Sugammadex, a neuromuscular blockade reversal agent, causes neurona apoptosis in primary cultures. Int J Med Sci 10: 1278-1285.

26. Shimizu S, Eguchi Y, Kamiike W, Itoh Y, Hasegawa J, et al. (1996) Induction of apoptosis as well as necrosis by hypoxia and predominant prevention of apoptosis by Bcl-2 and Bcl-XL. Cancer Res 56: 2161-2166. 